\title{
Correlation of Vertical Dimension of Occlusion with the Length of Fingers in Different Ethnicity and Gender in Nepal
}

\author{
Ayusha Bajracharya ${ }^{1}$, Kanchana Shrestha ${ }^{2}$, Shyam Maharjan ${ }^{3}$, Suraj RB Mathema ${ }^{4}$
}

\begin{abstract}
Aim and objective: To compare and correlate the vertical dimension of occlusion (VDO) with the length of fingers in different gender and ethnicity in the Nepalese population.

Materials and methods: The study was done on 115 dental students in Kathmandu valley. The study included 41 male and 74 female samples among which 59 were Aryan and 56 were of Mongolian ethnicity. Measurement of VDO was done from the base of the nose to the base of the chin and measurement of finger length was done from the tip of the finger to the distal-most point closest to the metacarpophalangeal crease using a modified digital Vernier caliper. All the data were recorded and statistically analyzed.

Results: The VDO was positively correlated with all the fingers of the left and right hand with $p$ values $<0.05$. The mean VDO of the male was $4.28 \mathrm{~mm}$ higher than that of the female. The mean VDO among Aryans was $2.43 \mathrm{~mm}$ lower than that of Mongolians. The highest correlation of VDO in Aryans and Mongolians was seen with the length of the thumb. This correlation was statistically significant with a $p$ value $<0.05$.

Conclusion: The study showed that the VDO was positively correlated with the length of fingers. There was a positive association between the VDO and gender as well as ethnicity. A proportional relationship exists between VDO and the length of thumb irrespective of gender and ethnicity. Keywords: Ethnicity, Gender, Length of finger, Vertical dimension of occlusion.

International Journal of Prosthodontics and Restorative Dentistry (2021): 10.5005/jp-journals-10019-1301
\end{abstract}

\section{INTRODUCTION}

The vertical dimension of occlusion (VDO) is the distance between two selected anatomic or marked points when teeth are in maximal intercuspal position (GPT-9). ${ }^{1}$ Vertical dimension of occlusion has significant functional, esthetic, physiological, and psychological roles in a patient. ${ }^{2}$ Determination of the accurate vertical dimension is an important step in making a prosthesis with adequate esthetics and functional contacts that are harmonious with the existing masticatory muscles and temporomandibular joints. ${ }^{3}$ This distance tend to alter with loss of teeth and has to be established within the range of the patient's adaptability and acceptability. ${ }^{4}$

Studies have shown the use of various physiological and mechanical methods for the determination of vertical dimension through pre- and post-extraction records. ${ }^{5}$ These methods are either tedious, time-consuming, requiring special instruments, or expose patients to radiation. ${ }^{6}$ The determination of an acceptable VDO is dependent upon the clinical judgment, skill, and experience of the dentist.

Hand and wrist have been used to assess the growth of dentofacial structures as a skeletal maturity indicator. ${ }^{7}$ Studies done by Leonardo Da Vinci have shown a relationship between many proportions of the face with the length of the thumb. ${ }^{8}$ This theory has been supported by studies that have investigated the hypothesis that the VDO might be predicted by the craniofacial $^{9}$ and body measurements like the length of different fingers. A review of the literature reveals that Caucasian and Asian characteristics used in numerous craniofacial and body measurements may be inadequate for application to different racial or ethnic groups. Sociocultural and racial variables have a definite influence. ${ }^{10}$

This study was aimed to assess the correlation between VDO and finger length based on gender and ethnicity in a limited sample

\footnotetext{
${ }^{1-4}$ Department of Prosthodontics and Maxillofacial Prosthesis, People's Dental College and Hospital Pvt. Ltd., Kathmandu, Nepal
}

Corresponding Author: Ayusha Bajracharya, Department of Prosthodontics and Maxillofacial Prosthesis, People's Dental College and Hospital Pvt. Ltd., Kathmandu, Nepal, Phone: +91 9845409968, e-mail: ayushabjr@gmail.com

How to cite this article: Bajracharya A, Shrestha K, Maharjan S, et al. Correlation of Vertical Dimension of Occlusion with the Length of Fingers in Different Ethnicity and Gender in Nepal. Int J Prosthodont Restor Dent 2021;11(1):16-21.

Source of support: Nil

Conflict of interest: None

of the Nepalese population. This study will provide preliminary data to correlate with data obtained from other populations and help in further research to obtain an easy and precise measurement of VDO through fingers.

\section{Materials and Methods}

The study was conducted on 115 dental students of different dental colleges in Kathmandu Valley viz. People's Dental College and Hospital, Kantipur Dental College Teaching Hospital and Research Center, and Nepal Medical College-College of Dental Surgery. Ethical clearance was taken from Institutional Review Board, Institute of Medicine (IOM) Tribhuvan University.

\section{Sample Size Calculation}

The sample size was calculated from a similar article done in the Nepalese population by Basnet et al. ${ }^{11}$ 
Mean VDO among Mongoloid population of Nepal $=64.60$.

Standard deviation (sd1) of VDO among Mongoloid population of Nepal $=4.48$

Mean VDO among the Aryan population of Nepal $=67.93$

Standard deviation (sd2) of VDO among Mongoloid population of Nepal $=5.02$

Mean difference $=3.33$

Calculated effect size $=0.701$

Level of significance $\left(Z_{\alpha / 2}\right)=5 \%$

Power of the study to detect the difference between groups $\left(Z_{\beta}\right)=90 \%$

Sample size calculation formula $(n)$

$$
=\frac{\left[2 \sigma^{2}\left(Z_{\beta}+Z_{\alpha / 2}\right)^{2}\right]}{(\text { Effect size })^{2}},
$$

$\sigma^{2}$ is pooled std. deviation which is calculated

$$
=\frac{\left(n_{1}-1\right) \mathrm{sd}_{1}^{2}+\left(n_{2}-1\right) \mathrm{sd}_{2}^{2}}{\left(n_{1}+n_{2}-2\right)}
$$

After applying this formula, for two-sided, the required sample size per group (ethnicity) $=43$

Estimated sample size is $43+43=86$

By amplifying the sample size by $10 \%$ for measuring errors and $10 \%$ for non-response, the final estimated sample size is $86+8.6+$ $8.6=103.2$ or rounding it to 104 , i.e., 52 Aryans and 52 Mongolians. A total of 115 students were included in the study.

The duration of the study was 1 year. Among the study group, 59 were Aryan and 56 of them were Mongolians, 41 were males and 74 were females. Informed consent was taken with the dental students. Students with complete natural dentition and class 1 molar relationship within the age 19-30 years were included in the study. Subjects with previous orthodontic treatment or orthognathic surgery, extensive prosthesis, periodontally compromised teeth, malocclusion, abnormality of the finger were excluded from the study.

To obtain VDO measurements, a digital Vernier caliper (Kingsmart 6 inch LCD, Simba Pte Ltd) with the accuracy of $\pm 0.01 \mathrm{~mm}$ was used. The lower tip of the digital Vernier caliper was modified with the help of self-cure acrylic (DPI RR cold cure, Uttarakhand, India) to form a flat base for the chin to rest. This allowed for precise positioning of the caliper over the hard chin bone with varying soft tissue thickness in the horizontal and vertical plane. The reading surface of the caliper was covered with paper (Fig. 1).

Purposive sampling was done and those students who met the inclusion criteria were selected. The serial number was provided to each sample without considering gender and ethnicity. Samples were given proforma to fill in to acquire their personal information like name, gender, and ethnicity. Measurements were made based on their serial number. This information was categorized only when all the sample collection was completed to control bias.

Subjects were asked to stand straight. The position of the head was adjusted such that it was at a right angle to the floor with their mouth closed. The subjects were then asked to bite their teeth in occlusion with lips and cheek in a relaxed position without any expression. The occlusion was checked visually by retracting cheeks in the molar region.

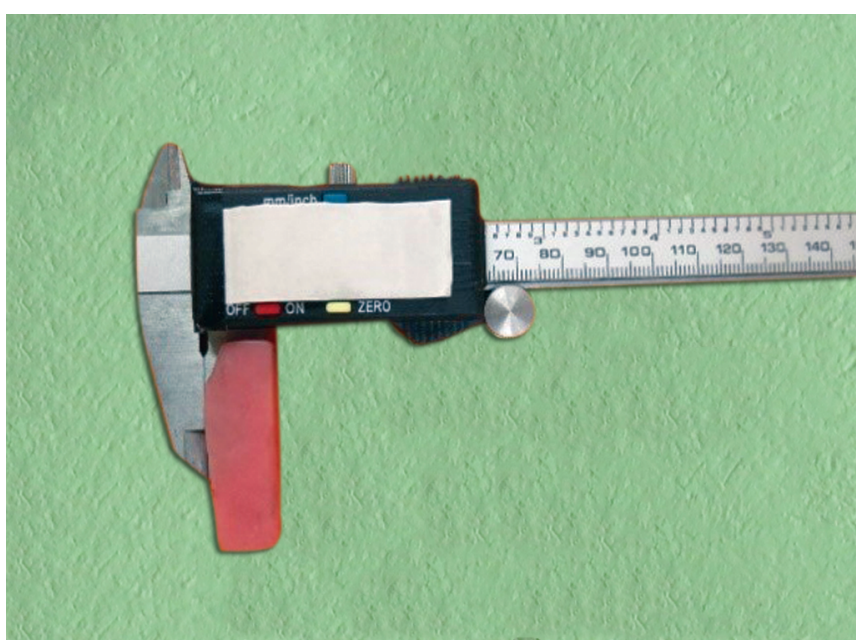

Fig. 1: Modified digital caliper with the reading surface covered with paper

Measurement was taken from the subnasion point at the base of the nose to the chin until the hard base of the bone was felt (Fig. 2). A modified digital Vernier caliper was used with a paper covering the digital reading. After the point of measurement was fixed, then only the display screen was uncovered (Fig. 3) to measure the length. A photograph of the reading was taken first then only the measurement was noted down. This highly reduced the measurement bias. Photograph of each sample with the measurement of VDO was clicked before the caliper reading was recorded. Total three readings of VDO were recorded.

For measurement of finger length, the hand was put flat on the table with the palm facing upward. Measurement was done from the tip of the finger to the point closest to the most distal metacarpophalangeal crease of all the fingers of both the right and left hand according to the serial number of the sample (Figs 4 and 5).

The data were entered, edited, and coded in Microsoft excel and were analyzed with the help of SPSS version 17.0. The continuous study variables like VDO and length of fingers and age were summarized with the help of mean and standard deviation. The categorical variables like race and gender were expressed in frequency and percentages. Association between VDO and length of fingers was checked with the help of Pearson's correlation coefficient and coefficient of determination. Similarly, the same correlation was checked in different races and gender. The level of significance was set at $5 \%$.

\section{Results}

Table 1 shows an association between VDO and gender. Statistically significant with $p$ value $<0.001$ implying that gender is associated with VDO. The mean value of VDO was $4.28 \mathrm{~mm}$ more in males than in females. Table 2 shows an association between VDO and ethnicity. A statistically significant $p$ value of 0.005 implies that Mongolians have significantly higher VDO than Aryans. The mean difference in VDO was $2.42 \mathrm{~mm}$. Table 3 shows a correlation of VDO with finger length. This was checked with the help of Pearson's correlation coefficient and coefficient of determination. The highest value of correlation coefficient $(R)$ was seen between right and left thumb with values of 0.559 and 0.470 .

Table 4 shows a correlation of VDO with finger length among Aryans. Bivariate correlation showed that the highest correlation with VDO in Aryan was seen with the right and left thumb with a 


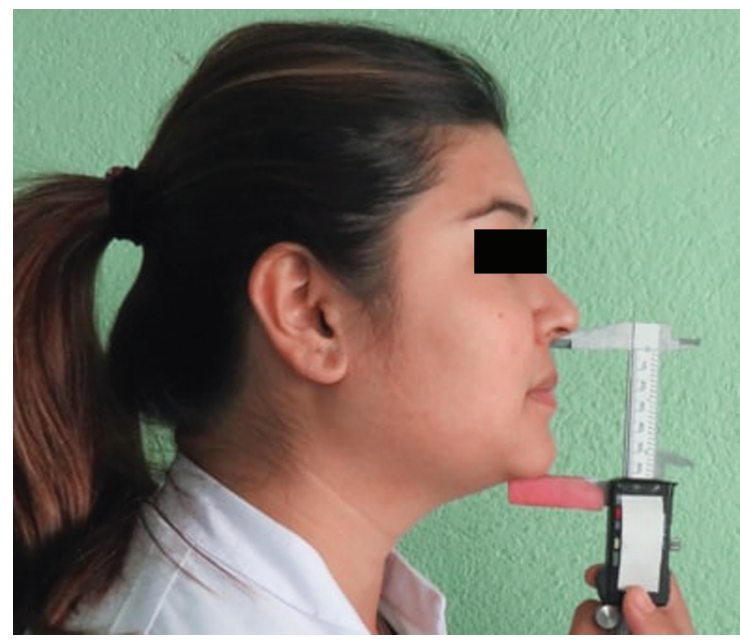

Fig. 2: Fixing the measuring point for VDO with caliper surface covered

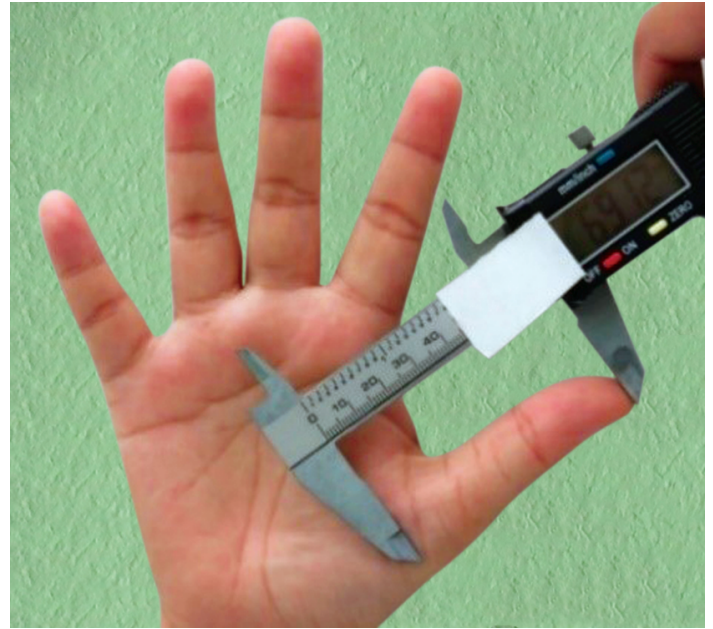

Fig. 4: Measurement of right finger length

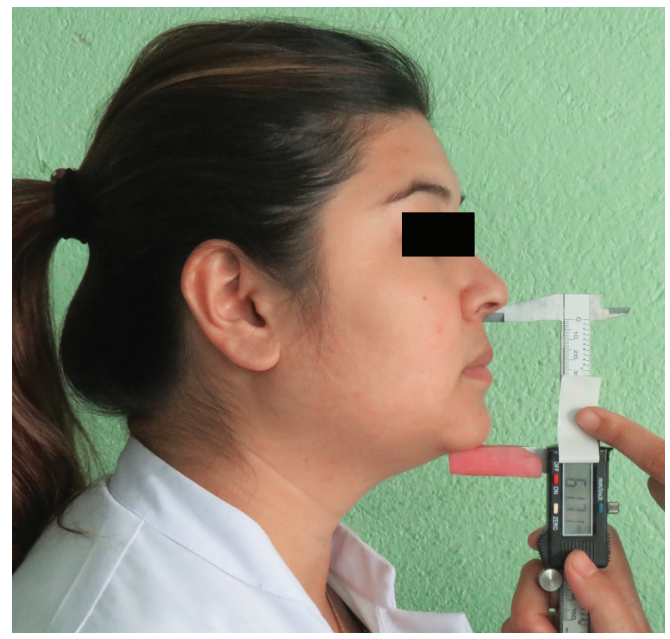

Fig. 3: Measurement of the VDO after uncovering the reading surface

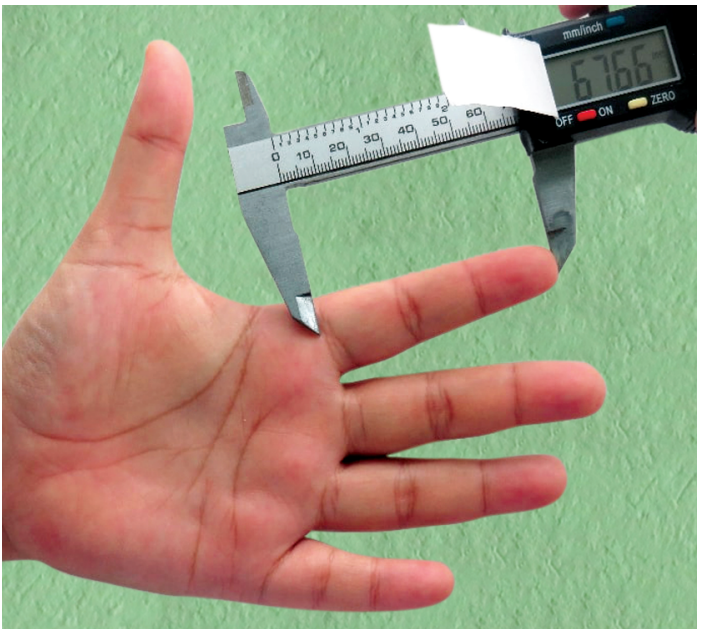

Fig. 5: Measurement of left finger length

Table 1: Association between gender and VDO $(n=115)$

\begin{tabular}{llllllll}
\hline & & & \multicolumn{3}{c}{$95 \%$ Cl of mean diff. } \\
\cline { 4 - 6 } & Male $($ mean $\pm S D)$ & $\begin{array}{l}\text { Female } \\
(\text { mean } \pm S D)\end{array}$ & Mean diff. & Lower & Upper & $p$ value \\
\hline VDO & $64.57 \pm 4.68$ & $60.28 \pm 3.90$ & 4.28 & 2.670 & 5.907 & $<0.001^{*}$ \\
\hline
\end{tabular}

*Statistically significant

Table 2: Association between ethnicity and VDO $(n=115)$

\begin{tabular}{lllllll}
\hline & & & \multicolumn{3}{c}{$95 \%$ Cl of mean diff } \\
\cline { 5 - 7 } & Aryan $($ mean $\pm S D)$ & $\begin{array}{l}\text { Mongolian } \\
(\text { mean } \pm S D)\end{array}$ & Mean diff. & Lower & Upper & $p$ value \\
\hline VDO & $60.63 \pm 4.49$ & $63.06 \pm 4.53$ & -2.426 & -4.096 & -0.756 & $\mathbf{0 . 0 0 5 ^ { * }}$ \\
\hline
\end{tabular}

*Statistically significant

value of $R$ being 0.543 and 0.424 , respectively. This correlation was statistically significant with a $p$ value $<0.05$.

Table 5 shows a correlation of VDO with finger length among Mongolians. Among Mongolians, all the fingers of the right and left hand showed a positive significant correlation with VDO with a $p$ value $<0.05$. The highest correlation of VDO was seen with right and left thumb with an $R$ value of 0.573 and 0.517 , respectively. Table 6 shows a correlation of VDO with finger length among males. The length of the right thumb was only significantly associated with VDO with an $R$ value of 0.379 and a $p$ value of 0.014 in males. Table 7 shows a correlation of VDO with finger length among females. The length of the right and left thumb was significantly associated with VDO with an $R$ value of 0.461 and 0.360 , respectively, in females. Figure 6 shows scatter diagrams showing the relationship of VDO with right and left thumb. The scatter diagram shows that the best fit line is upward sloping indicating there is positive correlation between Vertical Dimension at occlusion and length of left and right thumbs with $R^{2}$ values of 0.383 and 0.221 , respectively. 
Table 3: Correlation of VDO with fingers length $(n=115)$

\begin{tabular}{|c|c|c|c|}
\hline Variables & Fingers & Correlation coefficient $(R)$ & $p$ value \\
\hline \multirow[t]{11}{*}{ Vertical dimension at occlusion } & Age & -0.011 & 0.907 \\
\hline & Right thumb & 0.559 & $<0.001^{*}$ \\
\hline & Left thumb & 0.470 & $<0.001^{*}$ \\
\hline & Right forefinger & 0.227 & $0.015^{*}$ \\
\hline & Left forefinger & 0.258 & $0.005^{*}$ \\
\hline & Right middle finger & 0.271 & $0.003^{*}$ \\
\hline & Left middle finger & 0.250 & $0.007^{*}$ \\
\hline & Right ring finger & 0.293 & $0.001^{*}$ \\
\hline & Left ring finger & 0.287 & $0.002 *$ \\
\hline & Right little finger & 0.314 & $0.001 *$ \\
\hline & Left little finger & 0.287 & $0.002^{*}$ \\
\hline
\end{tabular}

*Statistically significant

Table 4: Correlation of VDO with finger length among Aryans

\begin{tabular}{|c|c|c|c|}
\hline Variables & Fingers & Correlation coefficient (R) & $p$ value \\
\hline \multirow[t]{10}{*}{ Vertical dimension at occlusion } & Right thumb & 0.543 & $<0.001^{*}$ \\
\hline & Left thumb & 0.424 & $0.001^{*}$ \\
\hline & Right forefinger & 0.143 & 0.281 \\
\hline & Left forefinger & 0.138 & 0.296 \\
\hline & Right middle finger & 0.237 & 0.071 \\
\hline & Left middle finger & 0.151 & 0.253 \\
\hline & Right ring finger & 0.256 & 0.051 \\
\hline & Left ring finger & 0.187 & 0.156 \\
\hline & Right little finger & 0.232 & 0.07 \\
\hline & Left little finger & 0.224 & 0.089 \\
\hline
\end{tabular}

*Statistically significant

Table 5: Correlation of VDO with finger length among Mongolians

\begin{tabular}{|c|c|c|c|}
\hline Variables & Fingers & Correlation coefficient ( $R$ ) & $p$ value \\
\hline \multirow[t]{10}{*}{ Vertical dimension at occlusion } & Right thumb & 0.573 & $<0.001^{*}$ \\
\hline & Left thumb & 0.517 & $<0.001^{*}$ \\
\hline & Right forefinger & 0.312 & $0.019^{*}$ \\
\hline & Left forefinger & 0.387 & $0.003^{*}$ \\
\hline & Right middle finger & 0.282 & $0.035^{*}$ \\
\hline & Left middle finger & 0.327 & $0.014^{*}$ \\
\hline & Right ring finger & 0.339 & $0.011^{*}$ \\
\hline & Left ring finger & 0.363 & $0.006^{*}$ \\
\hline & Right little finger & 0.354 & $0.007^{*}$ \\
\hline & Left little finger & 0.345 & $0.009^{*}$ \\
\hline
\end{tabular}

*Statistically significant

\section{Discussion}

Vertical dimension of occlusion can be affected by tooth eruption, TMJ, gonial angle, and length of the mandibular ramus. ${ }^{12}$ These factors are affected by race, nutritional intake, gender, and types of food intake during growth and developmental period. Leonardo da Vinci and $\mathrm{McGee}^{13}$ correlated VDO with other anthropometric measurements. The relationship between VDO and length of fingers was studied taking into account that the growth of body parts takes place in proportion to each other.
This present study was done on 115 dental students of Aryan and Mongolian ethnicity. Aryan and Mongolian are the two most common ethnic groups among the Nepalese population. Considering the age criteria of the study, the sample consisted of only dental students studying in different dental colleges in Kathmandu valley. Of them 41 were males and 74 were females among which 59 were Aryan and 56 were of Mongolian ethnicity. The result of this study has shown that the VDO was positively correlated with all the fingers of the left and right hand with $p$ values $<0.05$. The highest value of correlation coefficient $(R)$ was 
Table 6: Correlation of VDO with finger length among males

\begin{tabular}{llll}
\hline Variables & Fingers & Correlation coefficient (R) & $p$ value \\
\hline Vertical dimension at occlusion & Right thumb & 0.379 & $0.014^{*}$ \\
& Left thumb & 0.248 & 0.118 \\
& Right forefinger & 0.073 & 0.648 \\
& Left forefinger & 0.127 & 0.430 \\
& Right middle finger & 0.107 & 0.506 \\
& Left middle finger & 0.013 & 0.934 \\
& Right ring finger & 0.168 & 0.294 \\
& Left ring finger & 0.141 & 0.381 \\
& Right little finger & 0.119 & 0.458 \\
& Left little finger & 0.056 & 0.730 \\
\hline
\end{tabular}

*Statistically significant

Table 7: Correlation of VDO with finger length among females

\begin{tabular}{|c|c|c|c|}
\hline Variables & Fingers & Correlation coefficient (R) & $p$ value \\
\hline \multirow[t]{10}{*}{ Vertical dimension at occlusion } & Right thumb & 0.461 & $<0.001^{*}$ \\
\hline & Left thumb & 0.360 & $0.002^{*}$ \\
\hline & Right forefinger & 0.066 & 0.574 \\
\hline & Left forefinger & 0.056 & 0.638 \\
\hline & Right middle finger & 0.111 & 0.345 \\
\hline & Left middle finger & 0.117 & 0.321 \\
\hline & Right ring finger & 0.058 & 0.626 \\
\hline & Left ring finger & 0.072 & 0.541 \\
\hline & Right little finger & 0.182 & 0.120 \\
\hline & Left little finger & 0.133 & 0.257 \\
\hline
\end{tabular}

*Statistically significant
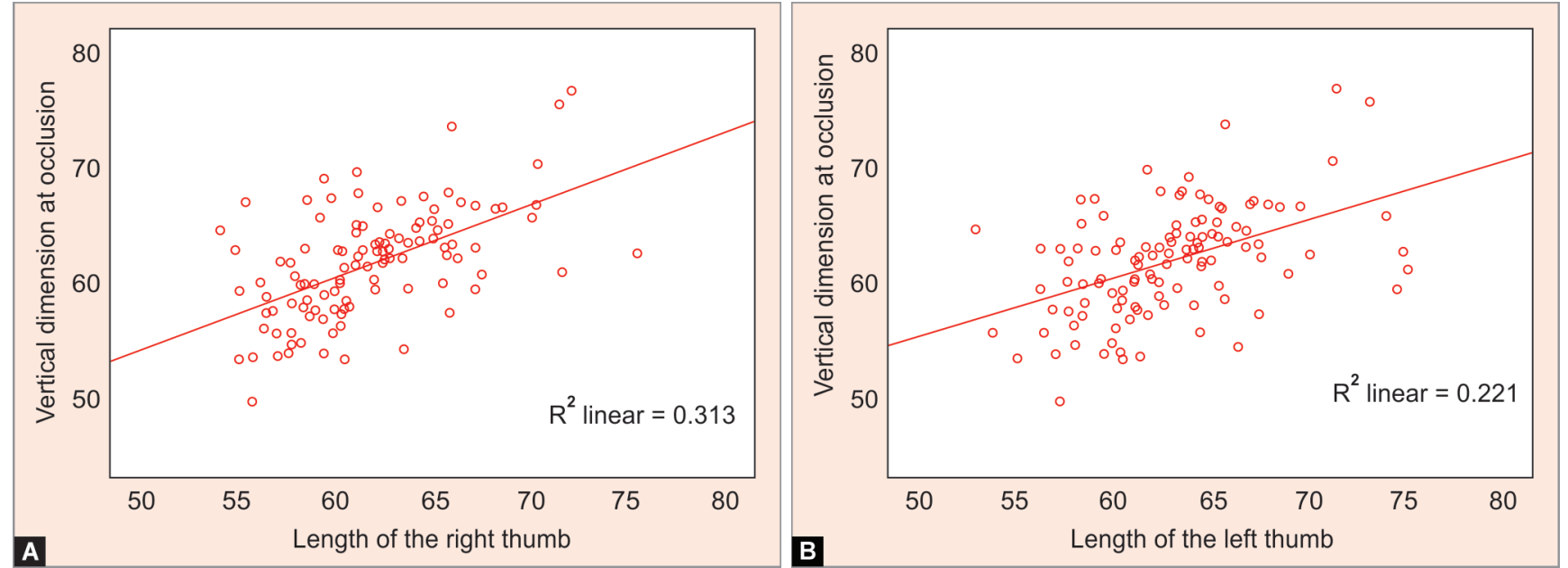

Figs $6 A$ and $B$ : Scatter diagrams to show the relationship of VDO with right and left thumb

seen between the right and left thumb with values of 0.559 and 0.470 , respectively.

The present study showed a difference in gender with higher values for VDO and length of fingers in males compared to females. The length of the right thumb was significantly associated with VDO with an $R$ value of 0.379 and a $p$ value of 0.014 in males and an $R$ value of 0.461 and 0.360 , respectively, in females.

Ethnicity has been observed to have an influence on VDO and the length of the finger. Researches by Oguz ${ }^{8}$ and Misch ${ }^{14}$ carried out a similar study among Caucasians. Among the two ethnicities in this study, Mongolians showed significantly higher VDO than Aryans. The mean VDO for Aryans was $2.43 \mathrm{~mm}$ lower than that of Mongolians. This difference may be considered to be due to different facial proportions in these ethnic groups. The result of this study has also been supported by a study done by Basnet et al. ${ }^{9}$ In the study, Aryans had a mean VDO of $63.89 \pm 6.29 \mathrm{~mm}$ and Mongolian had $65.86 \pm 3.61$ which signified that Mongoloids have longer VDO in comparison to Aryans. ${ }^{9}$ 
Bivariate correlation of VDO with a length of fingers among the Aryan and Mongolian ethnic group was tested. The highest correlation with VDO in Aryans was seen with the right and left thumb with a value of $R$ being 0.543 and 0.424 , respectively, and in Mongolian was seen with right and left thumb with an $R$ value of 0.573 and 0.517 , respectively. Ginting et al. in 2016 showed positive correlation between finger length and the VDO in Batak Toba Ethnic group. ${ }^{15}$ Alhajj ${ }^{16}$ and Hussain and Yazdanie ${ }^{17}$ also found significant correlations in-between VDO and index finger length in both genders. In studies done by Munshi et al. ${ }^{18}$ in Pakistani population and Rahmi et al. ${ }^{19}$ in Indonesian subraces such as Deutro-Malay, Proto-Malay, and Indonesian Chinese subraces, correlation of VDO was positive and strongest with the length of little finger. Similar result was seen in a study done by Krishnamurthy in 2021 Chennai where positive correlation was observed between VDO and length of index and little finger. ${ }^{20}$ Significant correlation between VDO and thumb length has been observed by Sajjan et al. ${ }^{21}$ and Teng et al. in undergraduate Mongoloid students in Indonesia. ${ }^{22}$

This study has shown a correlation between VDO and length of thumb irrespective of different gender and ethnicity. Thus, the length of thumb can be suggested to estimate the VDO and this method can be used as an alternative measure to obtain the lost VDO in this population. Limitation of the study was a limited sample. To validate these findings, further studies should be carried out comprising larger dentulous samples including other ethnic groups for appropriate regression equations for universal acceptance. However, the operator should keep in mind that VDO is the result of a musculoskeletal balance. The correct VDO can be better described as a range instead of as a fixed point. Therefore, to evaluate the VDO, a mixed-method should be adopted at all the stages of treatment to maximize the benefits and minimize harm to the stomatognathic system.

\section{Conclusion}

This study has shown that regardless of racial differences and sexual dimorphism between males and females, a proportional relationship exists between VDO and the length of the thumb. Therefore, in this population, the length of thumb could be suggested to estimate the VDO and it could be used as an alternative measure to obtain the lost VDO.

\section{References}

1. The glossary of prosthodontic terms. J Prosthe Dentis 2017;117(5):e1e105. DOI: 10.1016/j.prosdent.2016.12.001.

2. McCord J, Grant A. Identification of complete denture problems: a summary. British dental journal 2000;189(3):128-134. DOI: 10.1038/ sj.bdj.4800703.

3. Wyatt C, Zarb GA. Treatment outcomes of patients with implantsupported fixed partial prostheses. Int J Oral Maxillofac Implants 1998;13(2):204-211.

4. Heartwell CMR, Arthur O. Syllabus of complete denture. 4th edn., 1986. p. 573.

5. Alhajj M, Khalifa N, Abduo J, et al. Determination of occlusal vertical dimension for complete dentures patients: an updated review. J Oral Rehabilitat 2017;44(11):896-907. DOI: 10.1111/joor.12522.
6. Turrell A. Clinical assessment of vertical dimension. J Prosthe Dentis 2006;96(2):79-83. DOI: 10.1016/j.prosdent.2006.05.015.

7. Fishman LS. Radiographic evaluation of skeletal maturation: a clinically oriented method based on hand-wrist films. The Angle Orthodontist 1982;52(2):88-112. DOI: 10.1043/0003-3219(1982)0522.0. $\mathrm{CO} ; 2$.

8. Oguz $\mathrm{O}$. The proportion of the face in younger adults using the thumb rule of Leonardo da Vinci. Surg Radiol Anat 1996;18(2):111-114. DOI: 10.1007/BF01795228.

9. Basnet BB, Singh RK, Parajuli PK, et al. Correlation between facial measurements and occlusal vertical dimension: an anthropometric study in two ethnic groups of Nepal. Int J Dent Sci Res 2014;2(6):171174. DOI: 10.12691/ijdsr-2-6-13.

10. Ladda R, Bhandari AJ, Kasat VO, et al. A new technique to determine vertical dimension of occlusion from anthropometric measurements of fingers. Indian J Dent Res 2013;24(3):316. DOI: 10.4103/09709290.117993.

11. Basnet BB, Parajuli PK, Singh RK, et al. An anthropometric study to evaluate the correlation between the occlusal vertical dimension and length of the thumb. Clin Cosme Investigat Dentis 2015;7:33-39. DOI: 10.2147/CCIDE.S75872.

12. Spear F, Kinzer F. Approaches to vertical dimension. Adv Esthet Interdiscip Dent 2006;2(3):2-12.

13. McGee GF. Use of facial measurements in determining vertical dimension. J Am Dent Associat 1947;35(5):342-350. DOI: 10.14219/ jada.archive.1947.0361.

14. Misch CE. Clinical indications for altering vertical dimension of occlusion. Objective vs subjective methods for determining vertical dimension of occlusion. Quintessence International (Berlin, Germany: 1985) 2000;31(4):280-282.

15. Ginting R, Abidin T, Dennis D, et al. Convertion values of vertical dimension occlusion height to length of right hand fingers among batak toba ethnic. IOSR J Dent Med Sci 2016;15(6):40-46.

16. Alhajj MNA. Assessment of different measurement methods in predicting the occlusal vertical dimension among different populations in Sudanese and Yemeni. Univers Khartoum 2016. p. 127.

17. Hussain S, Yazdanie N. Correlation of the vertical dimension of occlusion with anthropometric measurement of index finger. JPDA 2019;28(03):109. DOI: 10.25301/JPDA.283.108.

18. Munshi MSM, Shah MU, Shaikh MI, et al. Role of anthropometric measurements in determining occlusal vertical dimension. Pakistan Oral Dent J 2020;40(2).

19. Rahmi E, Hidayati H, Suprianto K, et al. Correlation between length of little finger and occlusal vertical dimension in Indonesian subraces. Padjadjaran J Dentis 2020;32(1):33-38. DOI: 10.24198/pjd. vol32no1.24044.

20. Krishnamurthy $D$, Shanmuganathan DN. An anthropometric correlation of vertical dimension of occlusion, length of finger and linear ear length among dentulous patients-an observational study. Eur J Mole Clin Med 2021;8(3):549-557.

21. Sajjan MS, Eachempati P, Dhall R, et al. An anthropometric study to evaluate the correlation of vertical dimension at rest and length of thumb: a multi-national, multi-centre pilot study. J Indian Prosthodon Soc 2020;20(4):402. DOI: 10.4103/jips.jips_313_20.

22. Teng GL, Subrata G, Ardan R. The comparison between the length of vertical dimension of occlusion and the length of thumb on undergraduate Mongoloid students. Padjadjaran J Dentis 2011;23(1):39-45. DOI: 10.24198/pjd.vol23no1. 14060. 